\title{
Rings of definable scalars of Verma modules
}

\author{
Sonia l'Innocente *and Mike Prest \\ University of Manchester
}

November 21, 2006

\begin{abstract}
Let $M$ be a Verma module over the Lie algebra, $\operatorname{sl}_{2}(k)$, of trace zero $2 \times 2$ matrices over the algebraically closed field $k$. We show that the ring, $R_{M}$, of definable scalars of $M$ is a von Neumann regular ring and that the canonical map from $U\left(\mathrm{sl}_{2}(k)\right)$ to $R_{M}$ is an epimorphism of rings. We also describe the Ziegler closure of $M$. The proofs make use of ideas from the model theory of modules.
\end{abstract}

\section{Introduction}

If $R$ is any ring and $M$ any (right) $R$-module then the ring of definable scalars of $M$, denoted $R_{M}$, is the ring of pp-definable maps from $M$ to itself. Here "definable" means definable in the natural first-order language for $R$-modules and "pp-definable" means definable by a positive primitive formula. The operations in $R_{M}$ are composition and pointwise addition. Of course for every $r \in R$ the operation, multiplication by $r$, is pp-definable so there is a natural ring homomorphism $R \rightarrow R_{M}$ and it is easily checked that $R_{M}$ is a subring of the biendomorphism ring, $\operatorname{End}(\operatorname{End}(M) M)$, of the $R$-module $M$.

There are various alternative, but equivalent, ways of defining this ring (see, [3], [6]). For instance, we may localise the forgetful functor $\left({ }_{R} R,-\right) \in$ $(R$-mod, $\mathbf{A b})$, where $R$-mod denotes the category of finitely presented left $R$ modules, at a certain hereditary torsion theory determined by $M$ (precisely, at the, finite type, torsion theory generated by those finitely presented functors $F$ in $(R$-mod, $\mathbf{A b})$ which satisfy $(F, M \otimes-)=0)$. Then $R_{M}$ is the endomorphism ring of this localised functor.

Ivo Herzog proved [6] a number of rather remarkable results about the ring of definable scalars for the set of finite-dimensional representations of the Lie algebra $\mathrm{sl}_{2}(k)$ (that is for $M$ being the direct sum of all these modules). We

*and Department of Mathematics and Computer Science, University of Camerino, Italy. The first author was supported by a MATHLOGAPS Marie Curie Fellowship 
may ask what happens if we replace the set of finite-dimensional modules by the set of Verma modules. It even makes sense to investigate the ring of definable scalars for a single Verma module: since the module is infinite-dimensional, the situation is non-trivial (for a finite-dimensional module the ring of definable scalars simply is the biendomorphism ring $[3,3.6])$.

Let $k$ be a field of characteristic zero, which we also assume to be algebraically closed (though certainly not all of what we say needs this), and consider the Lie algebra, $\mathrm{sl}_{2}=\mathrm{sl}_{2}(k)$, of those $2 \times 2$ matrices over $k$ with trace 0 . A $k$-basis of $\operatorname{sl}_{2}(k)$ is given by $x=\left(\begin{array}{ll}0 & 1 \\ 0 & 0\end{array}\right), y=\left(\begin{array}{ll}0 & 0 \\ 1 & 0\end{array}\right), h=\left(\begin{array}{cc}1 & 0 \\ 0 & -1\end{array}\right)$.

The Lie bracket, which in terms of the algebra of matrices is $[u, v]=u v-v u$, is given by $[h, x]=2 x,[h, y]=-2 y,[x, y]=h$. The category of representations of any Lie algebra $L$ is equivalent to the category of left modules over its universal enveloping algebra $U(L)$ which, in the case $L=\mathrm{sl}_{2}(k)$, is $k\langle x, y, h\rangle /\langle h x-x h=2 x, h y-y h=-2 y, x y-y x=h\rangle$. We will not distinguish between representations of $L$ and left $U(L)$-modules.

Every finite-dimensional module over $U=U\left(\mathrm{sl}_{2}\right)$ is a direct sum of simple modules and, for every natural number $n$, there is, up to isomorphism, exactly one of these, $L(n)$, of dimension $n+1$ (see, e.g., [7, Section 6.1]). As a $k$ vectorspace, $L(n)$ has a basis $m_{-n}, m_{-n+2}, \ldots, m_{n-2}, m_{n}$ of eigenvectors of $h$ with the action of $\mathrm{sl}_{2}$ (and hence of $U\left(\mathrm{sl}_{2}\right)$ ) determined by $h m_{k}=k m_{k}, x m_{k}=$ $k(n+k-1) m_{k+2}$ (with $m_{k+2}$ interpreted as 0 when $k+2>n$ ) and $y m_{k}=$ $m_{k-2}$ (with a similar comment if $k-2<-n$ ) (see, e.g., [7, Section 7]). That completely describes the finite-dimensional representations but there are many infinite-dimensional simple modules: including the Verma modules, which we define now.

For $\lambda \in k$ set $M=M(\lambda)=U\left(\mathrm{sl}_{2}\right) / I_{\lambda}$, where $I_{\lambda}=U x+U(h-\lambda)$, to be the corresponding Verma module. This is the $U$-module generated by an element $m_{\lambda}$ (the image of $1 \in U$ ) with relations $x m_{\lambda}=0, h m_{\lambda}=\lambda m_{\lambda}$. Then a $k$-basis for $M(\lambda)$ is $m_{\lambda}, m_{\lambda-2}=y m_{\lambda}, \ldots, m_{\lambda-2 i}=y^{i} m_{\lambda}, \ldots(i \geq 0)$ and one may check that $h m_{\lambda-2 i}=(\lambda-2 i) m_{\lambda-2 i}$. Therefore, setting $M_{\mu}=\{m \in M: h m=\mu m\}$ to be the $\mu$-eigenspace for the action of $h$ on $M$, one has $M=\bigoplus_{i \geq 0} M_{\lambda-2 i}$ with each $M_{\lambda-2 i} 1$-dimensional. The action of $x$ is easily computed to be $x m_{\lambda-2 i}=$ $i(\lambda-n+1) m_{\lambda-2 i+2}$ (with $m_{\lambda+2}$ being interpreted as 0$)$.

If $\lambda$ is not a natural number then $M(\lambda)$ is a simple module, also often denoted $L(\lambda)$, and if $\lambda$ is a natural number, $n$, then $M(n)$ is a module of length two with composition factors $L(n)$ and $L(-n-2)$ (see, e.g., [8, p. 75]). We use the fact, $[4,7.18]$ that the endomorphism rings are trivial: $\operatorname{End}(M(\lambda))=k$ (for, consider the, commuting, actions on $m_{\lambda}$ of an endomorphism and $h$ ).

For background on representations of Lie algebras see [4] and [7] and for model theory of modules see [9] and [15] or, when it is complete, [12] (the last especially for results described here as "folklore"). 


\section{Definable subgroups of Verma modules}

If $R$ is any ring then the usual model-theoretic language for $R$-modules has symbols with which to express the addition (in a module) and the scalar multiplication (of each element of $R$ on a module), as well as a symbol for the zero element of a module. So the basic ("atomic") formulas are just linear equations: $\sum_{i=1}^{n} x_{i} r_{i}=0$ where the $x_{i}$ are variables and the $r_{i}$ elements of $R$. The pp ("positive primitive") formulas are those obtained from conjuctions of such equations (i.e. from systems of linear equations) by existentially quantifying out some of the variables. Formally, such a formula, $\phi$ ( or $\phi\left(x_{1}, \ldots, x_{k}\right)$ if we wish to show its unquantified variables) has the shape $\exists x_{k+1}, \ldots, x_{n}\left(\bigwedge_{j=1}^{m} \sum_{i=1}^{n} x_{i} r_{i j}=0\right)$. What such a formula defines is the projection, to the first $k$ coordinates, of the solution set to a system of $R$-linear equations. Clearly the set so defined is a group. If $\phi$ is a pp formula with just one free variable and if $M$ is a module then the solution set to $\phi$ in $M$ is denoted $\phi(M)$ and is a typical pp-definable subgroup of $M$ : note that this is closed under the action of endomorphisms of the $R$-module $M$. In particular, any pp-definable subgroup of a Verma module is a $k$-vectorspace.

Lemma 2.1 Suppose that $M$ is a finitely presented module over an arbitrary ring $R$ and that $\operatorname{End}(M)=k$ is a field (or just a division ring). Then every finite-dimensional $k$-subspace of $M$ is pp-definable.

This is "folklore": we indicate the proof. Since a finite sum of pp-definable subgroups is easily seen to be pp-definable, it is enough to prove this for 1dimensional subspaces of $M$. So let $a \in M, a \neq 0$. If $c_{1}, \ldots, c_{n}$ is a generating set for $M$ and if $\sum_{i=1}^{n} c_{i} r_{i j}=0(j=1, \ldots, m)$ is a generating set of relations for $M$, and if $a=\sum_{i=1}^{n} c_{i} s_{i}$ then let $\phi(x)$ be the pp formula $\exists x_{1}, \ldots, x_{n}\left(\left(\bigwedge_{j=1}^{m} \sum_{i=1}^{n} x_{i} r_{i j}=0\right) \wedge x=\sum_{i=1}^{n} x_{i} s_{i}\right)$ (the symbol $\wedge$ should be read as "and" and $\Lambda$ is to $\Lambda$ as $\sum$ is to + ). It is quite easy to check that $\phi(M)=a k$, as required. In fact, this last point may be generalised, as follows.

First we define the pp-type of an $n$-tuple $\bar{a}=\left(a_{1}, \ldots, a_{n}\right)$ o f elements from a module $M$ to be the set, $\mathrm{pp}^{M}(\bar{a})$, of pp formulas $\phi$, with $n$ free variables, such that $\bar{a} \in \phi(M)$. It is the case (see $[9,8.4]$ ) that if $M$ is finitely presented then there is a pp formula $\phi_{0}$ in $\mathrm{pp}^{M}(\bar{a})$ which generates this pp-type in the sense that for every module $N$ (in particular for $M$ ) we have $\phi_{0}(N) \leq \phi(N)$ for every $\phi \in \operatorname{pp}^{M}(\bar{a})$.

Lemma 2.2 Suppose that $M$ is a finitely presented module over an arbitrary ring $R$ and that $\operatorname{End}(M)=k$ is a field (or just a division ring). Let $\bar{a}=$ $\left(a_{1}, \ldots, a_{n}\right)$ be an $n$-tuple from $M$. Let $\phi_{0}$ be a pp formula which generates $\operatorname{pp}^{M}(\bar{a})$. Then $\phi_{0}(M)$ is 1-dimensional over $k$.

Proof. Suppose that $\bar{b} \in \phi_{0}(M)$. By $[9,8.5]$ there is an endomorphism of $M$ taking $\bar{a}$ to $\bar{b}$. By assumption, that endomorphism is multiplication by an 
element of $k$.

When dealing with $\mathrm{sl}_{2}$-modules we will use $u, v$, rather than $x, y$, for variables, for obvious reasons (and we will deal with left, rather than right, modules).

Proposition 2.3 Let $M(\lambda), \lambda \notin \mathbb{N}$ be a Verma module over $\operatorname{sl}_{2}(k)$. Then $M(\lambda)$ has neither the ascending nor descending chain condition on pp-definable subgroups. In particular $M$ is not pure-injective ("pure-injective" means injective over pure embeddings, where an embedding $A \leq B$ of modules is said to be pure if, for every pp formula $\phi(x)$, we have $\phi(A)=A \cap \phi(B)$, equivalently if the induced morphism $\left(A \otimes_{R}-\right) \rightarrow\left(B \otimes_{R}-\right)$ of functors from left $R$-modules is monic).

Proof. The images of successive powers of $y$ give an infinite descending chain and the annihilators of successive powers of $x$ give an infinite ascending chain. Since $M(\lambda)$ is countable-dimensional it is not, since the dcc fails, $\Sigma$-pureinjective (another piece of folklore, with a proof similar to that in $[9,3.1(\mathrm{c})]$ ), in particular it is not pure-injective.

Lemma 2.4 Let $M(\lambda)$ be as above. Every finite-dimensional subspace of $M(\lambda)$ is pp-definable. Furthermore, every cofinite-dimensional subspace, $W$, of $M(\lambda)$ which contains some $M_{\leq \lambda-2 i}=\bigoplus_{s=i}^{\infty} M_{\lambda-2 s}$ is pp-definable.

Proof. The first statement is by 2.1 .

For the second statement, first note that $M_{\leq \lambda-2 i}$ itself is pp-definable (by the formula which expresses the condition $\left.y^{i} \mid v\right)$. Then write $W$ as $M_{\leq \lambda-2 i} \oplus V$ where $V \leq M_{>\lambda-2 i}$. Thus $W$ is the sum of two pp-definable subgroups, hence is pp-definable.

The subgroups above do not exhaust the cofinite-dimensional pp-definable subgroups: consider, for example, the image of $1+y$. Of course, 'most' cofinitedimensional subspaces are not pp-definable, since there are too many of them - for instance, if $k$ is countable then they are uncountable in number whereas there are only countably many pp conditions with which to define subspaces.

We have the following further observations about the ring of definable scalars, $R_{\lambda}=R_{M_{\lambda}}$ of $M(\lambda)$.

First, if $W$ is any pp-definable subspace which has a pp-definable complement, $W^{\prime}$, then projection to $W$ with kernel $W^{\prime}$ is a pp-definable map (hence is in the ring of definable scalars). For, if $W=\phi(M)$ and $W^{\prime}=\psi(M)$ then let $\rho(u, v)$ be the formula $\phi(v) \wedge \exists w(\psi(w) \wedge u=v+w)$. Then the binary relation $\rho(M(\lambda)) \subseteq M^{2}$ clearly is this projection function on $M$.

Hence if $V$ is any finite-dimensional subspace of $M$ or if $V$ is any cofinitedimensional subspace which contains some $M_{\leq \lambda-2 n}$ then projection to $V$ is pp-definable.

There is also the following. 
Lemma 2.5 If $V$ is any finite-dimensional subspace of $M=M(\lambda)$ and $T$ : $V \longrightarrow V$ is any $k$-linear endomorphism of $V$ then there is a definable scalar, $s$, of $M(\lambda)$ such that $s \uparrow V=T$.

Proof. Choose a basis $a_{1}, \ldots, a_{n}$ for $V$. By 2.1 there are pp formulas $\phi_{i}$ such that $\phi_{i}(M)=\left\langle a_{i}\right\rangle$ (the vector subspace generated by $a_{i}$ ). For each $i$ let $\rho_{i}\left(u_{i}, v_{i}\right)$ be a pp formula which generates the pp-type of the pair $\left(a_{i}, T a_{i}\right)$ in $M$ (this exists by $[9,8.4])$. Let $\rho^{\prime}(u, v)$ be the pp formula $\exists u_{1}, \ldots, u_{n}, v_{1}, \ldots, v_{n}(u=$ $\left.\sum_{1}^{n} u_{i} \wedge \bigwedge_{1}^{n} \phi_{i}\left(u_{i}\right) \wedge \bigwedge_{1}^{n} \rho_{i}\left(u_{i}, v_{i}\right) \wedge v=\sum_{1}^{n} v_{i}\right)$. Clearly if $a \in V$ then $\rho^{\prime}(a, T a)$ holds and that the converse also holds may be checked using that each $\rho_{i}(M) \subseteq$ $M^{2}$ is, by 2.2 , 1-dimensional.

Let $\phi$ be a pp formula defining $V$ and let $\psi$ be a pp formula defining a complement, $W$, for $V$ in $M$ (such exists by 2.4). Finally let $\rho(u, v)$ be the formula $\exists z\left(\psi(z) \wedge \phi(u-z) \wedge \phi(v-z) \wedge \rho^{\prime}(u-z, v-z)\right)$. Clearly $\rho\left\lceil W=\operatorname{id}_{W}\right.$ and $\rho \uparrow V=T$, as required.

\section{Generalised Weyl Algebras}

Let $k$ be a field. The first Weyl algebra over $k$ is the algebra $A_{1}(k)=k\langle x, y$ : $y x-x y=1\rangle$. There are a number of important algebras with properties similar, to some degree, to those of $A_{1}(k)$. These were given a uniform treatment by Bavula ([1], also see [2]) and are referred to as "generalised Weyl algebras".

In order to define these generalised Weyl algebras start with the polynomial ring $k[H]$. Choose an automorphism, $\sigma$, of the ring $k[H]$ and a nonconstant polynomial $a=a(H) \in k[H]$. From this data define the algebra $k[H]\left\langle x, y: y x=a, x y=\sigma(a), b y=y \sigma(b), b x=x \sigma^{-1}(b),(b \in k[H])\right\rangle$ (in fact Bavula's definition is more general). Generalised Weyl algebras where the action of $\sigma$ is given by $\sigma(H)=H-1$ include the Weyl algebra itself (set $H=y x$ and take $a=H$ ) and all non-semisimple primitive factor rings of the universal enveloping algebra of $\operatorname{sl}_{2}(k)$ (see below).

Proposition 3.1 [13, 3.1] Let $R$ be a generalised Weyl algebra over a field of characteristic 0 with $\sigma(H)=H-1$. Then no finite length module, apart from 0 , is divisible.

Lemma 3.2 Suppose that $R$ is a generalised Weyl algebra over a field of characteristic 0 with $\sigma(H)=H-1$. Let $M$ be a simple $R$-module. Then $\bigcap\{M r$ : $r \in R, r \neq 0\}=0$.

Proof. We show that $V=\bigcap\{M r: r \in R, r \neq 0\}$ is a submodule of $M$. Let $a \in V$ and let $r \in R, r \neq 0$. It must be shown that ar $\in V$. Let $s \in R, s \neq 0$. Since $R$ is an Ore domain $R r \cap R s \neq 0$, say $r^{\prime} r=s^{\prime} s \neq 0$. Since $a \in V$ there is $a^{\prime} \in M$ with $a=a^{\prime} r^{\prime}$ and hence $a r=a^{\prime} r^{\prime} r=a s^{\prime} s$, so $a r \in M s$, as required. 
If $V$ is non-zero then $M=V$ is divisible, contradicting 3.1.

Lemma 3.3 Let $R$ be any ring and let $M$ be a finitely presented $R$-module. Suppose that $\operatorname{End}(M)=k$ is a field, that every pp-definable subgroup of $M$ is finitedimensional or cofinite-dimensional and that $\bigcap\{(M): \phi(M)$ is cofinite-dimensional $\}=$ 0. Then the lattice of pp-definable subgroups of $M$ is complemented (i.e. given $\phi$ pp there is $\psi$ pp with $M=\phi(M) \oplus \psi(M))$.

Proof. Suppose that $\phi$ is a pp formula such that $\phi(M)$ is finite-dimensional over $k$. Choose $\psi$ such that $\psi(M)$ is cofinite-dimensional and with $\phi(M) \cap \psi(M)=0$. Such exists since, otherwise, choose $\psi$ with $\psi(M)$ cofinite-dimensional and with $\operatorname{dim}_{k}(\phi(M) \cap \psi(M))$ of minimum dimension. By hypothesis there is $\psi^{\prime}$ with $\psi^{\prime}(M)$ cofinite-dimensional and $\psi^{\prime}(M) \ngtr \psi(M) \cap \phi(M)$. Then $\psi \wedge \psi^{\prime}$ is cofinitedimensional and its intersection with $\phi(M)$ is strictly smaller than $\phi(M) \cap \psi(M)$ - contradiction.

Now choose any $k$-vectorspace complement, $W$, of $\psi(M)$ in $M$ with $W \geq$ $\phi(M)$ and choose a complement, $W^{\prime}$, for $\phi(M)$ within $W$. By $2.1 W^{\prime}=\phi_{0}(M)$ for some $\phi_{0}$. Then $\psi(M) \oplus \phi_{0}(M)$ is a pp-definable complement for $\phi(M)$.

On the other hand, if $\phi(M)$ is cofinite-dimensional then, immediately from 2.1 , it has a pp-definable complement.

Then we use the following result from Herzog's paper.

Theorem 3.4 [6, pp. 254, 255] Let $R$ any ring and let $M$ be an $R$-module such that the lattice of pp-definable subgroups of $M$ is complemented. Then the ring of definable scalars, $R_{M}$, is von Neumann regular and $R \longrightarrow R_{M}$ is an epimorphism of rings.

Corollary 3.5 Suppose that $M$ is finitely presented, that $\operatorname{End}(M)=k$ is a field, that every pp-definable subgroup of $M$ is finite-or cofinite-dimensional and that $\bigcap\{\phi(M): \phi(M)$ is cofinite-dimensional $\}=0$. Then $R_{M}$ is von Neumann regular and $R \longrightarrow R_{M}$ is an epimorphism of rings.

\section{Rings of definable scalars of Verma modules}

We will show that the ring of definable scalars of any Verma module $M(\lambda)$ is von Neumann regular. By 3.4 it will be enough to show that every pp-definable subgroup of $M(\lambda)$ has a pp-definable complement.

A key observation is that the simple Verma module $M(\lambda)(\lambda \notin \mathbb{N})$ is a module over a generalised Weyl algebra, as follows.

Set $\mu=\lambda^{2}+2 \lambda$ and let $C=2 x y+2 y x+h^{2}$ be the Casimir element of $U=U\left(\mathrm{sl}_{2}\right)$. Computation shows that the actions of $x, y$ and $h$ on $M(\lambda)$ satisfy the relations: $x y=\frac{1}{4}\left(\mu-(h-2)^{2}-2(h-2)\right)$ and $y x=\frac{1}{4}\left(\mu-h^{2}-2 h\right)$, as well 
as those, $x h=(h-2) x$ and $y h=(h+2) h$, coming from $U$ itself. Furthermore, computing the action of $C$ on $m_{\lambda-2 i}$, one sees that $C=\mu$ on $M(\lambda)$.

Therefore $M(\lambda)$ is a faithful module over the primitive factor ring $U_{\mu}=$ $U /\langle C-\mu\rangle$ of $U$ (see $[4,8.4 .3]$ ). (It is the case, $[4,8.4 .4]$, that, as $\lambda$ varies over all of $k$, these are all the primitive factor rings of $U$. Observe also that $\lambda^{2}+2 \lambda=\nu^{2}+2 \nu$ iff $\nu=\lambda$ or $\nu=-\lambda-2$ so $M(-\lambda-2)$ also is a $U_{\mu}$-module.) We check that the rings $U_{\mu}$ are generalised Weyl algebras.

Denote by $\sigma$ the automorphism of the polynomial ring $k[h]$ given by $\sigma(h)=$ $h-2$ and let $a(h)$ be the polynomial $\left(-h^{2}-2 h+\mu\right) / 4$. Then $U_{\mu}=k[h]\langle x, y$ : $\left.x h=\sigma(h) x, y h=\sigma^{-1}(h) y, x y=a(h-2), y x=a(h)\right\rangle$ or, making the change of variable $H=h / 2, U_{\mu}=k[H]\left\langle x, y: x H=\sigma(H) x, y H=\sigma^{-1}(H) y, x y=\right.$ $a(H-1), y x=a(H)\rangle$ where now $\sigma$ is given by $\sigma(H)=H-1$ and $a(H)=$ $-H^{2}-H+\frac{\mu}{4}$. So this is even a generalised Weyl algebra of the special form where the automorphism is given by $\sigma(H)=H-1$.

We remark that, by $\left[1,3.2\right.$, Thm. 5], $U_{\mu}$ is hereditary and a simple ring iff the roots of $H^{2}+H-\frac{\mu}{4}$ do not differ by an integer. The roots of this polynomial are $\frac{\lambda}{2}$ and $-\frac{\lambda}{2}-1$, so the difference is $-\lambda-1$, which is an integer iff $\lambda \in \mathbb{Z}$. Thus $U_{\mu}$ is hereditary and simple if(f) $\lambda \notin \mathbb{Z}$.

Proposition 4.1 Suppose that $R$ is a generalised Weyl algebra with $\sigma(H)=$ $H-1$. Let $M$ be a simple $R$-module. Then the ring, $R_{M}$, of definable scalars of $M$ is von Neumann regular and the natural map $R \longrightarrow R_{M}$ is an epimorphism.

Proof. We apply 3.5. The finite/cofinite hypothesis is satisfied, by [13, 3.3] The intersection condition is by 3.2. (The non-zero subspaces $M r$ are cofinitedimensional by $[13,2.2,3.3]$.)

Corollary 4.2 Let $M=M(\lambda)$ be a Verma module for $\operatorname{sl}_{2}(k)$ ( $k$ algebraically closed of characteristic 0$)$. Then its ring of definable scalars, $R_{M}$, is a von Neumann regular ring and the natural map $R \longrightarrow R_{M}$ is an epimorphism of rings.

(The case where $\lambda \in \mathbb{N}$ follows since then $M(\lambda)$ is the direct sum of the finite-dimensional simple module $L(\lambda)$ and the Verma module $M(-\lambda-2)$, each of which has von Neumann regular ring of definable scalars (for the finitedimensional module this follows by $[3,3.6]$, which implies that the ring of definable scalars is the full, simple artinian, biendomorphism ring.))

\section{The Ziegler-closure of a Verma module}

The Ziegler spectrum, $\mathrm{Zg}_{R}$, of a ring $R$ is a topological space, introduced in [15], the points of which are the isomorphism classes of indecomposable pureinjective modules. A basis of open sets is given by those of the form $(\phi / \psi)=$ 
$\left\{N \in \mathrm{Zg}_{R}: \phi(N)>\psi(N)\right\}$ where $\phi$ and $\psi$ are pp formulas with $\phi(M) \geq \psi(M)$ for every module $M$. Its usefulness in the model theory of modules can be seen from [9] and some examples of its algebraic relevance are in [11].

Every module $M$ has a pure-injective hull - a minimal pure-injective module into which $M$ purely embeds. If $M$ is finitely presented with local endomorphism ring then, [5, p. 157], the pure-injective hull, $H(M)$, of $M$ is indecomposable, hence a point of $\mathrm{Zg}_{R}$.

Proposition 5.1 Let $M=M(\lambda), \lambda \notin \mathbb{N}$ be a Verma module over $\operatorname{sl}_{2}(k)$. Then the Ziegler-closure of the pure-injective hull, $H(M)$, of $M$ consists of $H(M)$ and $D_{\lambda}$ where $D_{\lambda}$ is the quotient division ring of (the noetherian domain) $U_{\lambda}$. The point $H(M)$ is isolated in its closure. Furthermore this closure also is homeomorphic to the Ziegler spectrum, $\mathrm{Zg}_{R_{M}}$, of the ring of definable scalars of $M$.

Proof. The first statement follows by [13, 3.7], the second is obvious (directly or since $D_{\lambda}$ clearly is a closed point). For the third we need the general fact that, if $M$ is a module over the von Neumann regular ring $S$, then the Ziegler-closure of $H(M)$ is that of $H(S / \operatorname{ann}(M))$ (see, e.g., [6, p. 255]). The last statement then follows by [10, Thm. 1(c), Cor. 9] since $R \longrightarrow R_{M}$ is an epimorphism.

It also follows from $[13,3.5]$ that $H(M(\lambda)) / M(\lambda) \simeq D_{\lambda}^{(\kappa)}$ for some (infinite by $[13,3.2])$ cardinal $\kappa$.

By 5.1 the Cantor-Bendixson rank of $\mathrm{Zg}_{R_{M}}$ is 1 (that is, once the isolated points are removed, there remain only points isolated in what remains). For the dimensions, Krull-Gabriel and m-, which appear in the next result, see, for instance, [11].

Corollary 5.2 Let $M=M(\lambda), \lambda \notin \mathbb{N}$, be a Verma module over $s l_{2}(k)$. Then $\mathrm{CB}\left(\mathrm{Zg}_{R_{M}}\right)=\mathrm{KG}\left(R_{M}\right)=\operatorname{mdim}\left(M_{R}\right)=1$.

Proof. The fact that the m-dimension of $M$ is 1 is immediate from the fact that every pp-definable subgroup is of finite or cofinite height in the lattice of pp-definable subgroups of $M$. That this equals the Krull-Gabriel of $R_{M}$ is then a consequence of 5.1 and the, already-mentioned, fact that the Ziegler-closures of $M$ and $R_{M}$ are homeomorphic and that the latter is, because $R_{M}$ is von Neumann regular, the closed subset of $\mathrm{Zg}_{U}$ corresponding to the category of $R_{M}$-modules. (It is also a consequence of a result of Trlifaj $[14,3.6]$ which says that semiartinian regular rings, which these are (see below), satisfy the isolation condition, plus Ziegler's result [15, 8.6], see [9, 10.19], that, in the presence of this condition, Cantor-Bendixson rank and m-dimension coincide).

We can say a little more about $R_{M}$ when $M$ is a Verma module. By 5.1, $D_{\lambda}$ is a (simple) $R_{M}$-module and hence, if $I=\operatorname{ann}_{R_{M}} D_{\lambda}$ then $R_{M} / I$ is a simple regular ring which is artinian (e.g. by 5.1). Also by 5.1 the only other simple $R_{M}$-module is $M$ itself. 
Proposition 5.3 Let $M=M(\lambda), \lambda \notin \mathbb{N}$, be a Verma module over $\operatorname{sl}_{2}(k)$ and let $R_{M}$ be its ring of definable scalars. Then $R_{M}$ is semiartinian.

Proof. Regard $R_{M}$ as a subring of $\operatorname{Biend}\left(M_{U}\right)=\operatorname{End}\left({ }_{k} M\right) \simeq \operatorname{End}\left({ }_{k} k^{\left(\aleph_{0}\right)}\right)$. The image of each element of $R_{M}$, being a pp-definable subgroup, is (see before 2.5) either finite-dimensional or cofinite-dimensional and all finite-dimensional subspaces of ${ }_{k} M$ occur as images, so clearly the socle of $R_{M}$ consists of those definable scalars which have finite-dimensional image. But this is exactly $I=$ $\operatorname{ann}_{R_{M}} D_{\lambda}$ and $R / I$ is artinian. Hence $R_{M}$ is, indeed, semiartinian.

We have left unresolved the analogue of Herzog's result. Namely, is the main result, 4.2, true for $M$ being the direct sum of all the Verma modules? (Arguably the correct analogue is the corresponding statement for a direct sum of modules of the form $M_{\lambda+n}$ where $\lambda$ is fixed and $n \in \mathbb{Z}$.)

\section{References}

[1] V. Bavula, Generalised Weyl algebras and their representations, Algebra and Analiz, 4(1) (1992), 75-97; English transl. in St. Petersburg Math. J., 4(1), (1993), 71-92.

[2] V. Bavula and F. van Oystaeyen, The simple modules of certain generalised crossed products, J. Algebra, 194 (1997), 521-566.

[3] K. Burke and M. Prest, Rings of definable scalars and biendomorphism rings, pp. 188-201 in Model Theory of Groups and Automorphism Groups, London Math. Soc. Lect. Note Ser., Vol. 244, Cambridge University Press, 1997.

[4] J. Dixmier, Algèbres Enveloppantes, Gauthier-Villars, 1974, Engl. transl. Enveloping Algebras, North-Holland, 1977.

[5] I. Herzog, The Auslander-Reiten translate, Contemp. Math., 130 (1992), 153-165.

[6] I. Herzog, The pseudo-finite dimensional representations of $s l(2, k)$, Selecta Mathematica, 7 (2001), 241-290.

[7] J. E. Humphreys, Introduction to Lie Algebras and Representation Theory, Graduate Texts in Math., Vol. 9, Springer, 1972.

[8] J. E. Humphreys, Highest weight modules for semisimple Lie algebras, pp. 72-103 in V. Dlab and P. Gabriel (Eds.) "Representation Theory I", Springer Lecture Notes in Mathematics, Vol. 831, 1980.

[9] M. Prest, Model Theory and Modules, London Math. Soc. Lecture Notes Ser. Vol. 130, Cambridge University Press, 1988. 
[10] M. Prest, Epimorphisms of rings, interpretations of modules and strictly wild algebras, Comm. Algebra, 24 (1996), 517-531.

[11] M. Prest, Topological and geometric aspects of the Ziegler spectrum, pp. 369-392 in H. Krause and C. M. Ringel (eds.), Infinite Length Modules, Birkhäuser, 2000.

[12] M. Prest, Purity, Spectra and Localisation, book in preparation.

[13] M. Prest and G. Puninski, Pure injective envelopes of finite length modules over a Generalised Weyl Algebra, J. Algebra, 251 (2002), 150-177.

[14] J. Trlifaj, Two problems of Ziegler and uniform modules over regular rings, pp. 373-383 in Abelian Groups and Modules, M. Dekker, New York, 1996.

[15] M. Ziegler, Model theory and modules, Ann. Pure Applied Logic, 26 (1984), 149-213. 